\title{
Tirela: an unusual asteroid family in the outer main belt
}

\author{
T. Mothé-Diniz ${ }^{1,2, \star}$ and D. Nesvorný ${ }^{2,3, \star \star}$ \\ 1 Observatório Nacional, Rua Gal. José Cristino, 77, 20921-400 Rio de Janeiro, Brasil \\ 2 UFRJ/Observatório do Valongo, Ladeira Pedro Antônio, 43, 20080-090 Rio de Janeiro, Brasil \\ e-mail: thais.mothe@astro.ufrj.br \\ ${ }^{3}$ Southwest Research Institute, 1050 Walnut St., Suite 400, Boulder, Colorado 80302, USA \\ e-mail: davidn@boulder.swri .edu
}

Received 29 May 2008 / Accepted 15 September 2008

\section{ABSTRACT}

\begin{abstract}
Context. Earlier visible spectroscopic classification of (1400) Tirela suggests that this object is a D-type. As the Tirela family has high eccentricities and inclinations, its parent-body could have been implanted in the main belt during the late heavy bombardment, a scenario recently proposed for some D-type asteroids. Spectra of other members of the family have not been available until now, except for colors from the Sloan Digital Sky Survey (filters $u, g, r, i$, and $z$ ) for some of them. These colors also suggest that the family has slopes similar to D-types.

Aims. Thus, we wanted to spectroscopically characterize the Tirela family. Due to the faintness of the objects, we used some relatively big telescopes in some observing runs.

Methods. We obtained visible spectra of 10 faint Tirela family members at telescopes TNG and GEMINI and the NIR spectrum of two members of the family at the IRTF telescope. These spectra were classified and comparisons were made with meteorites and minerals in an attempt to infer the mineralogical surface composition of the members.

Results. The Tirela family was found to be composed of Ld-type asteroids. Comparisons with minerals suggest that the combined visible+near-infrared spectrum of (1400) Tirela show some similarities to the spectrum of the pure mineral pigeonite. Conclusions. We conclude that the family is not a D-type, but comparisons with minerals suggest similarities between the absorptions found in Tirela with those found in the mineral pigeonite.
\end{abstract}

Key words. minor planets, asteroids

\section{Introduction}

Asteroid families are groups of asteroid fragments with similar orbits and spectra that represent remnants of large, collisionally disrupted asteroids (Hirayama 1918; Zappalà et al. 1995). Asteroid families are useful targets for spectroscopic studies because different fragments of a single asteroid family sample different parts of the disrupted parent body. By spectroscopically characterizing different fragments, we may learn about the composition of the parent body.

The original orbit of the parent body of the Tirela family has high eccentricity and inclination $\left(e_{\mathrm{p}}=0.20\right.$ and $\left.i_{\mathrm{p}}=16.8 \mathrm{deg}\right)$ and is located at the edge of the outer asteroid belt (proper semimajor axis $\left.a_{\mathrm{p}}=3.12 \mathrm{AU}\right)$. The Tirela family have been recently identified by Nesvorný et al. (2005). Its members have colors - obtained from the Sloan Digital Sky Survey, SDSS (Stoughton et al. 2002) - that suggest a steep spectral slope similar to 1400 Tirela, which was observed spectroscopically by the S3OS2 Survey, and classified as a D-type asteroid (Lazzaro et al. 2004). While other families have been extensively studied (Doressoundiram et al. 1998; Florczak et al. 1998, 1999; Lazzaro et al. 1999; Mothé-Diniz et al. 2001, 2005, 2008), very little spectroscopic information exists about the Tirela family.

\footnotetext{
* Author T. Mothé-Diniz was supported by the Conselho Nacional de Desenvolvimento Científico e Tecnológico-CNPq/Brasil.

$\star \star$ The work of D.N. was supported by the NASA's Planetary Geology and Geophysics program.
}

Recent studies (Levison et al. 2008) have shown that many D-type objects have been implanted in the asteroid belt from beyond $20 \mathrm{AU}$ at the time of the late heavy bombardment. This population of comets then suffered a very heavy collisional evolution, which should have produced families. We, therefore, have selected the Tirela family, based on previously available S3OS2 and Sloan data, as a D-type candidate family that may have formed during the collisional phase described by Levison et al. (2008).

This work represents an effort to spectroscopically characterize the faint Tirela family members, to confirm the taxonomic classification they belong to, and to eventually detect the presence of any absorption bands such as the silicate bands around 1.0 and $2.0 \mu \mathrm{m}$ or any aqueous alteration bands that could exist. In the next section we describe the observation processes in the three different telescopes used. In Sect. 3 we describe the characteristics of the spectra obtained, and discuss the possible mineralogical associations. Section 4 summarizes the conclusions of this work.

\section{Observations}

Due to its location in the main belt ( $\left.a_{\mathrm{P}} \simeq 3.12 \mathrm{AU}\right)$, the Tirela family has very faint members. For observing a considerable amount of its members, we needed many runs on different telescopes. Here we describe how each of the groups of observations was done in chronological order. 


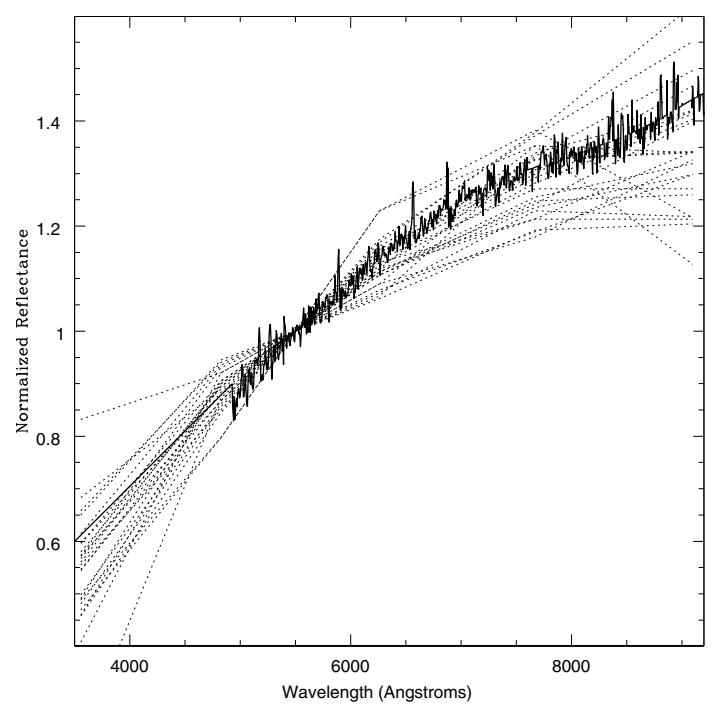

Fig. 1. Spectrum of (1400) Tirela (dark line) plotted together with the SDSS colors for Tirela family members (dotted lines).

\subsection{Infrared Telescope Facility - IRTF}

The near-infrared spectra of 2 Tirela family members were remotely obtained from the National Observatory with the $3.0-\mathrm{m}$ NASA Infrared Telescope Facility (IRTF) equipped with a $1024 \times 1024 \mathrm{InSb}$ array spectrograph $(\mathrm{SpeX})$, and located at the Mauna Kea Observatory in Hawaii on the night of June 20, 2006. We used a 0.8 arcsec slit oriented in the east-west direction. In the low-resolution prism-mode, this slit provides a resolution $R \sim 100$, with a spectrum covering the entire interval from $0.8-2.5 \mu \mathrm{m}$ in a single exposure. For the reduction of the data, we followed the standard procedures of flat-field correction and sky subtraction. The spectra were then extracted and calibrated in wavelength. Several spectra of solar analog stars were taken during the night (SA107684, SA110361, SA1121333, SA113276). These stars were used to produce normalized reflectance spectra of the asteroids. The final spectra presented in this paper are the ratio of the spectra of the asteroids, with the star SA 107 684, which was the star with more close airmass to that of the asteroids. Divisions by other solar analogs give similar final spectra, but with more noise. The error bars are not plotted, but the errors propagated through the reduction are usually less than the scatter in the data, so we assume that the scatter in the data is the best estimate of the uncertainties of each measurement.

\subsection{Telescopio Nazionale Galileo - TNG}

The visible spectrum of two members of Tirela family, (9222) and (43343), were obtained at the 3.58-m Telescopio Nazionale Galileo (TNG) on December 16, 2006. The telescope was equipped with the DOLORES (Device Optimized for the LOw RESolution) focal reducer, installed at the Nasmyth B focus of the TNG, with a $1.0^{\prime \prime}$ slit, and the Grism LR-R. This grism has a dispersion of $2.61 \AA / \mathrm{px}$, central wavelength at $740 \mathrm{~nm}$, and an wavelength interval from $447-1007 \mathrm{~nm}$, with a $R=714$. Flatfield, dark, and Ar-lamp calibration files were obtained at the beginning of the night, as an standard procedure at the TNG. For correction of the solar flux at the asteroids, the solar analog stars SA98-978, SA102-1081 and SA115-271 were also observed.

The science data and solar analogs were reduced using the package IRAF, following the standard procedures of flat-fielding, de-biasing, extraction, and wavelength calibration of spectra. Every spectra was then divided by every solar analog and the final spectra were compared. As expected, the closer the solar analog is in airmass to the asteroid, the smaller the noise in the final spectrum.

\subsection{GEMINI}

With the GEMINI 8.0-m telescopes, North and South, we obtained good $\mathrm{S} / \mathrm{N}$ data for asteroids (13150), (31704), (44443), (60157), (68147), (80279), and (84829). Observations were performed with the two Gemini Multi-Object Spectrographs (GMOS)(Hook et al. 2004), one at each GEMINI telescope, under the programs GS2007A-C-6 and GN-2007B-Q-67. Observations of program GS2007A-C-6 were made in classical mode, while those of program GN2007A-C-6 were made in queue mode. Both cameras have a pixel size of $0.0727^{\prime \prime} /$ pixel with a field of view of $330 \times 330 \mathrm{arcsec}^{2}$. The spectrographs were used in the long-slit mode, with a slit of 1.5". The filter GG455G0305 was used for the observations, making the wavelength interval $\geq 460 \mathrm{~nm}$ and the effective wavelength $\geq 550 \mathrm{~nm}$. This filter was used with the grating R150+G5306 for Gemini North, and the identical grating R150+G5326 for Gemini South, which yields a simultaneous coverage of $1071 \mathrm{~nm}$, with an $R=631$ and the Blaze wavelength is at $717 \mu \mathrm{m}$. Both GMOS-N and GMOS-S have significant fringing in the red (from $0.7 \mu \mathrm{m}$ on). To minimize these effects, for each science object we obtained an even number of spectra, pairs of spectra in two positions of the slit, and performed subtractions B-A. Calibration images of Flat and Arc were obtained via the Gemini Facility Calibration Unit (GCAL), with the same configuration as the science data. After the whole sequence of every object was concluded, three flatfield images and one arc were taken for calibration. The lamp available for arc calibration of optical spectra is the CuAr.

The reduction of these data was made using the standard Gemini IRAF package, with the sub-package GMOS. Biases were combined with the task "gbias", flats were combined using the task "gsflat", and science targets were reduced with task "gsreduce", which subtracts off the bias, mosaics the three detectors of GMOS, interpolates across the chip gaps for science data, and applies flat field correction. Then the CuAr images were also reduced with "gsreduce". For these arcs, the wavelength calibration was established with the task "gswavelength", and the science spectra were further transformed by applying the task "gstransform". Then the sky subtraction was done with "gsskysub", and the pairs of spectra A and B were subtracted, so that we obtained one positive and one negative spectrum. We then extracted all the negative and the positive spectra for each target. The same process was used for the solar analog stars, with the exception of the subtraction A-B. For the division of the asteroid spectra by the solar analogs, all spectra of each science target and solar analog were summed up. The final spectrum of each asteroid was then re-binned to match the same points of the star. The spectra were then rationed by the solar analog spectrum and normalized to unity at $0.55 \mu \mathrm{m}$.

In Table 1 we present the details of the observation of the targets, such as UT date, exposure times, phase angle, apparent magnitude, and the solar analog used for removing the solar flux from the asteroid.

\section{Spectroscopic characteristics}

We applied the algorithm of Bus \& Binzel (2002b) to taxonomically characterize our nine visible spectra, and again the 
Table 1. Observational details for asteroid spectra of Tirela Family members presented in this paper.

\begin{tabular}{|c|c|c|c|c|c|c|c|c|}
\hline$\overline{\text { Asteroid }}$ & $\begin{array}{l}\text { Spectral range/ } \\
\text { Telescope }\end{array}$ & $\overline{\text { UT date }}$ & $\begin{array}{c}\text { Exposure } \\
\text { (s) }\end{array}$ & $\begin{array}{l}\text { Mean } \\
\text { airmass }\end{array}$ & $\begin{array}{r}\text { Phase angle } \\
\text { (deg) }\end{array}$ & Mag. (V) & $\begin{array}{l}\text { Solar } \\
\text { Analog }\end{array}$ & Class \\
\hline (1400) Tirela & NIR/IRTF & 20-Jun.-2006 & 120 & 1.23 & 13.8 & 15.9 & SA107684 & $\overline{\mathrm{Ld}}$ \\
\hline (9222) 1995YM & Vis/TNG & 16-Dec.-2006 & 1500 & 1.07 & 4.8 & 16.3 & SA115271 & B \\
\hline (13150) Paolotesi & Vis/GEMINI $_{\mathrm{S}}$ & 15-Mar.-2007 & $6 \times 300$ & 1.01 & 18.8 & 18.36 & SA104335 & $\mathrm{Ld}$ \\
\hline (31704) 1999JZ44 & Vis/GEMINI $_{N}$ & 3-Jan.-2008 & $4 \times 250$ & 1.04 & 14.6 & 19.4 & SA92501 & $\mathrm{Ld} / \mathrm{D}$ \\
\hline (43343) 2000RY81 & Vis/TNG & 16-Dec.-2006 & 1500 & 1.02 & 7.7 & 16.3 & SA115271 & $\mathrm{Ld}$ \\
\hline (44443) 1998UY19 & Vis/GEMINI $_{S}$ & 17-Mar.-007 & $4 \times 210$ & 1.03 & 22.2 & 16.6 & SA104335 & $\mathrm{Sa} / \mathrm{Sl}$ \\
\hline (60157) 1999UT23 & Vis/GEMINIS & 17-Mar.-2007 & $6 \times 250$ & 1.03 & 8.5 & 17.9 & SA104335 & $\mathrm{Ld}$ \\
\hline (68147) 2001AW44 & Vis/GEMINI $_{S}$ & 17-Mar.-2007 & $6 \times 210$ & 1.08 & 4.9 & 17.5 & SA104335 & $\mathrm{Ld} / \mathrm{L}$ \\
\hline (80279) 1999XP33 & Vis/GEMINIS & 17-Mar.-2007 & $8 \times 260$ & 1.78 & 25.4 & 19.0 & SA104335 & $\mathrm{Ld} / \mathrm{A}$ \\
\hline (84829) 2003AN & Vis/GEMINI $_{N}$ & 16-Nov.-2007 & $4 \times 250$ & 1.27 & 9.9 & 18.5 & SA98682 & $\mathrm{Ld}$ \\
\hline (97768) 2000JP69 & NIR/IRTF & 20-Jun.-2006 & $4 \times 120$ & 1.25 & 20.5 & 17.6 & SA107684 & ? \\
\hline
\end{tabular}

GEMINI $_{S}$ stands for observations made with GEMINI South, while GEMINI ${ }_{N}$ for those made at GEMINI North telescope.

For the objects which still do not have a name, the first column shows the provisory designation together with the number of the asteroid.
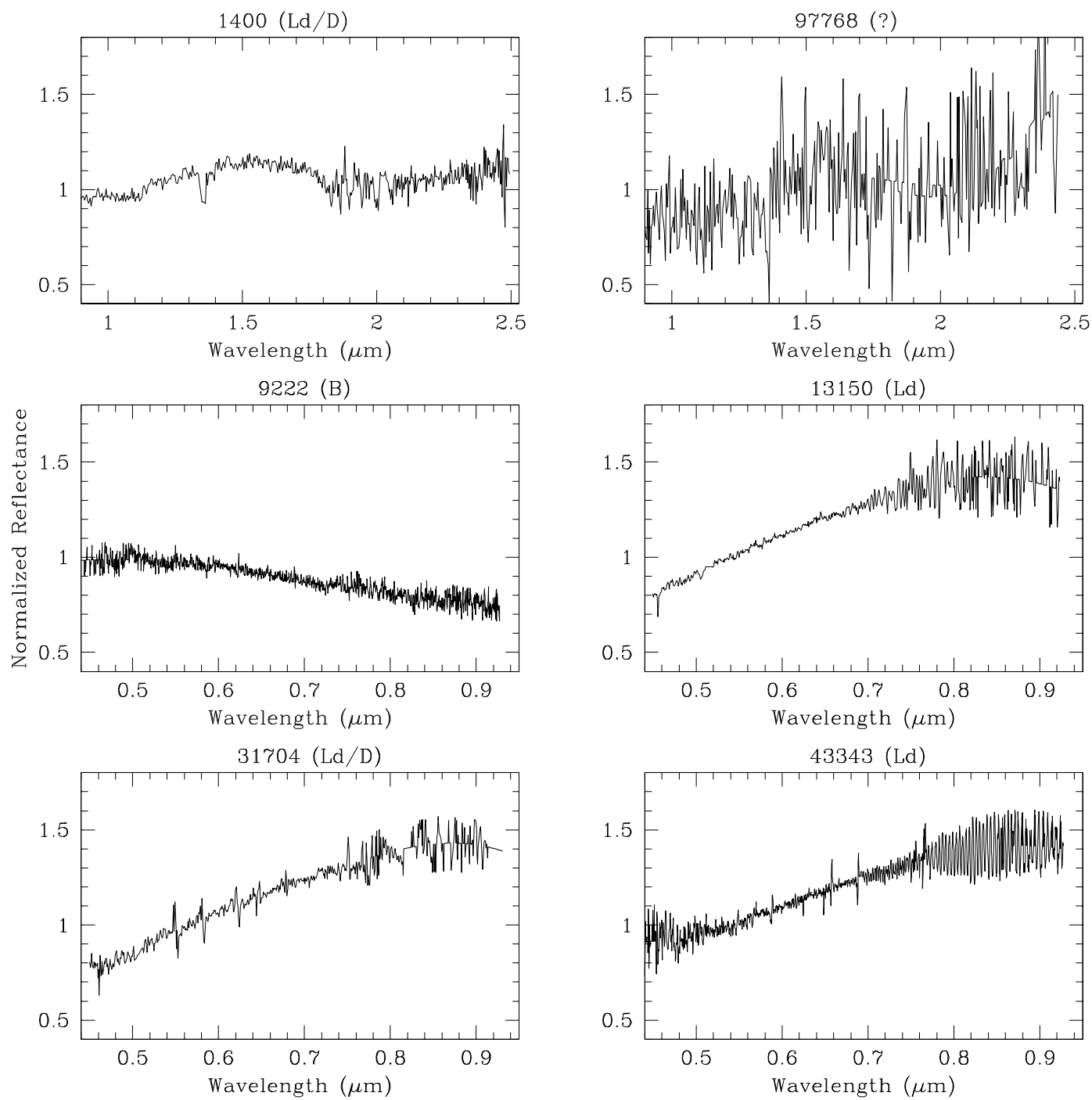

Fig. 2. Spectra of the objects observed in this study. The visible spectra were normalized to unity at $0.55 \mu \mathrm{m}$, while the NIR spectra were normalized at $1.2 \mu \mathrm{m}$. The visible spectrum of (1400) Tirela, taken from Lazzaro et al. (2004) is given in Fig. 3 below.

previously observed spectrum of (1400) Tirela. The main result of our observations was that none of the asteroid members of Tirela family could be classified as a D-type as a first choice. Eight members, including (1400) Tirela, have similar spectra, which match the characteristics described by Bus \& Binzel (2002a) for the Ld class, one belongs to the S-complex (Sl, Sa), and one was classified as B-type. This taxonomic classification was made by using the algorithm for taxonomic classification of Bus (Bus \& Binzel 2002a). The object (97768) 2000 JP69 could not be classified taxonomically since we only had its NIR spectrum. The spectrum of (1400) Tirela had previously been classified as D-type (Lazzaro et al. 2004), and it is in fact intermediate 

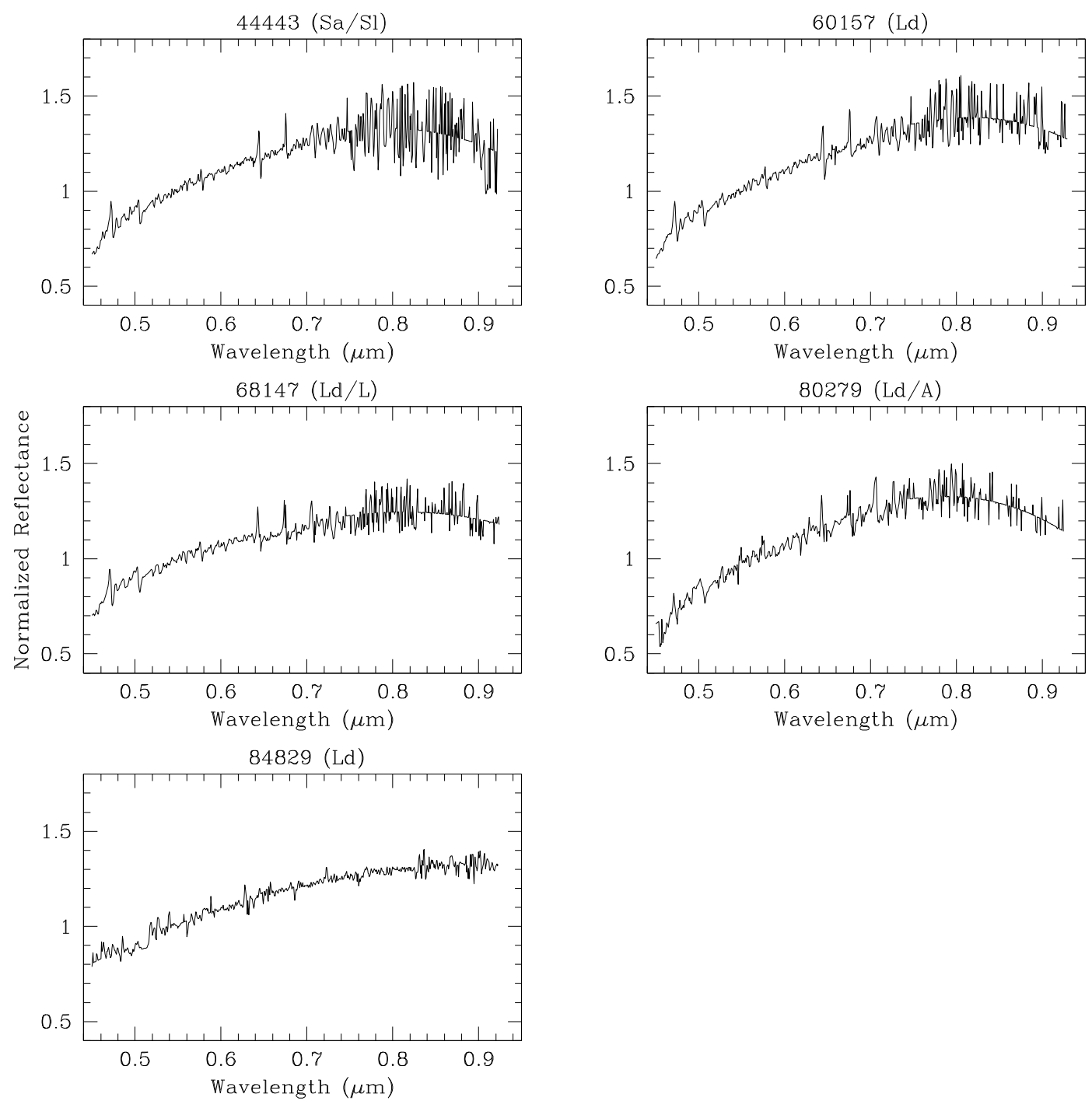

Fig. 2. Continued.

between a D and an Ld-type. Analysis of the spectroscopic characteristics alone shows that Ld-types differ from D-types because of their slight steeper spectrum under $0.75 \mu \mathrm{m}$ and for its flattened spectrum from $0.75 \mu \mathrm{m}$. Tirela's flatness after $0.75 \mu \mathrm{m}$, characteristic of Ld types, is less pronounced than for other Ld's observed in the family. In this work we re-classified the S3OS2 spectrum of (1400) Tirela as an Ld-type. A plot showing the comparison of the spectrum of Tirela with templates of $\mathrm{Ld}$ and D-types is shown in Fig. 3. These templates represent an average of the spectra of the respective Ld and D class objects of Bus \& Binzel (2002a) and are only used for visual reference.

The Ld, S1, and Sa classes found among Tirela family members were first defined by Bus \& Binzel (2002a), and since then a few works have been published associating them to some mineralogy. The $\mathrm{Sl}$ and Sa classes belong to the broader S class. Based on comparisons with meteorites in the spectroscopic range 0.44-1.65 $\mu \mathrm{m}$, Burbine \& Binzel (2002) associated Sl objects to LL-chondrites, as well as with pallasite- and brachinite-like achondrites, while Sa objects have spectra similar to metallic iron + olivine-rich silicates, H- and/or LL-chondrites. Burbine \& Binzel (2002) also associated the Ld class with CV or CK-like chondrites. As for the present S3OS2 and SMASSII databases, thirty asteroids are classified as Ld-types. Twenty-nine of them are spread in the region from 1.5-4.0 AU, apparently without

preference for groupings. One object, however, is a TransNeptunian object, with osculating elements for FK5/J2000.0 $a=285.3, e=0.99$, and $i=29.9$.

Except for the B-type, all the previous associations reveal that Tirela family members are silicate-rich bodies, possibly having suffered some amount of space weathering on their surfaces, so that they present some differences in slope. B-type objects are generally associated with the primitive $\mathrm{CI} / \mathrm{CM}$ chondrites and can also be associated to some CV chondrites (Carvano et al. 2003, and references therein). Thus, in the context of the other objects observed, the B-type asteroid could be considered as an interloper in this family.

\subsection{Attempt of mineralogical association}

Given the small amount of mineralogical information about the Ld class and given that we have the complete visible + nearinfrared (VNIR) spectrum of (1400) Tirela, we tried to find spectra of meteorites and/or minerals that present similarities in the $0.49-2.5 \mu \mathrm{m}$ to Tirela. The composite VNIR spectra were obtained by requiring that the reflectance of the NIR portion matched the visible spectrum at $0.8 \mu \mathrm{m}$. To this end, both spectra were smoothed with a spline fit, and the NIR spectrum was normalized by the ratio of the VIS to the NIR reflectance at $0.8 \mu \mathrm{m}$. 


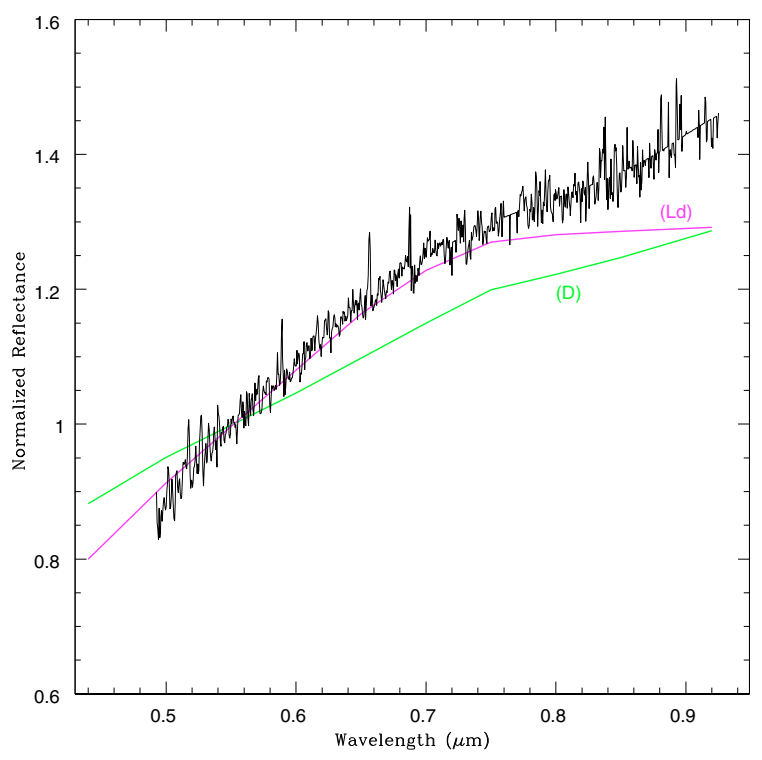

Fig. 3. Visible spectrum of (1400) Tirela compared to the templates of Ld and D taxonomic classes from Bus \& Binzel (2002a).

The resulting spectra were normalized to unity at $0.55 \mu \mathrm{m}$ by convention. As the family is located in the outer main belt, with a low probability of being the source of meteorites found on Earth, we did not expect to find any perfect match among the meteorites database, but clues to the possible surface mineralogy for this asteroid.

We compared the spectrum of Tirela with the RELAB (Pieters \& Hiroi 2004) database of meteorites and minerals. The comparison procedure was described in Mothé-Diniz \& Carvano (2005). Although Burbine \& Binzel (2002) associated some Ldtype asteroids with $\mathrm{CV}$ or $\mathrm{CK}$ meteorites, and L types with pallasite-like achondrites, metallic iron + pallasitic olivine, and hydrated spinel-bearing silicates, none of the 802 meteorites in the database had a spectrum similar to Tirela's. On the other hand, when comparing with the database of minerals available in RELAB and USGS (Clark et al. 2003), we found the spectrum of the mineral pigeonite from USGS (sample name pigeonite HS199.3B in splib05a) with positions of the 1 and $2 \mu \mathrm{m}$ bands in the reflectance spectrum very close to the positions of these bands in the spectrum of Tirela. Figure 4 shows the comparison between the visible and NIR spectra of Tirela and the spectrum of the mineral. In this plot we see that the depth (and position) of the $2.0 \mu \mathrm{m}$ bands matches very well, and also the whole region from $1.1 \mu \mathrm{m}$ to $2.5 \mu \mathrm{m}$. Also, the shape of both spectra is very similar in the visible region just before the $1.0 \mu \mathrm{m}$ band. The $1.0 \mu \mathrm{m}$ band itself, however, in the pigeonite has a much higher contrast than in the asteroid. This suggests some low-phase mineral at the surface reducing the contrast of the main-phase mineral of this asteroid. No other mineral in the mineral databases could match the absorptions found in the spectrum of Tirela.

Pigeonite is a monoclinic pyroxene, with general formula $(\mathrm{Ca}, \mathrm{Mg}, \mathrm{Fe})(\mathrm{Mg}, \mathrm{Fe}) \mathrm{Si}_{2} \mathrm{O}_{6}$. On Earth, most of the hightemperature metamorphic and igneous orthorhombic pyroxenes were originally pigeonite. On extra-terrestrial materials, pigeonites can be found as crystals in Martian and Lunar meteorites. It is also the primary mafic mineral of the differentiated Eucrites and Howardites meteorites. Small amounts of pigeonite can also be found in CAI inclusions in some chondrites, like $\mathrm{CV}$ or $\mathrm{CM}$. This is an interesting finding, since pigeonite, as we

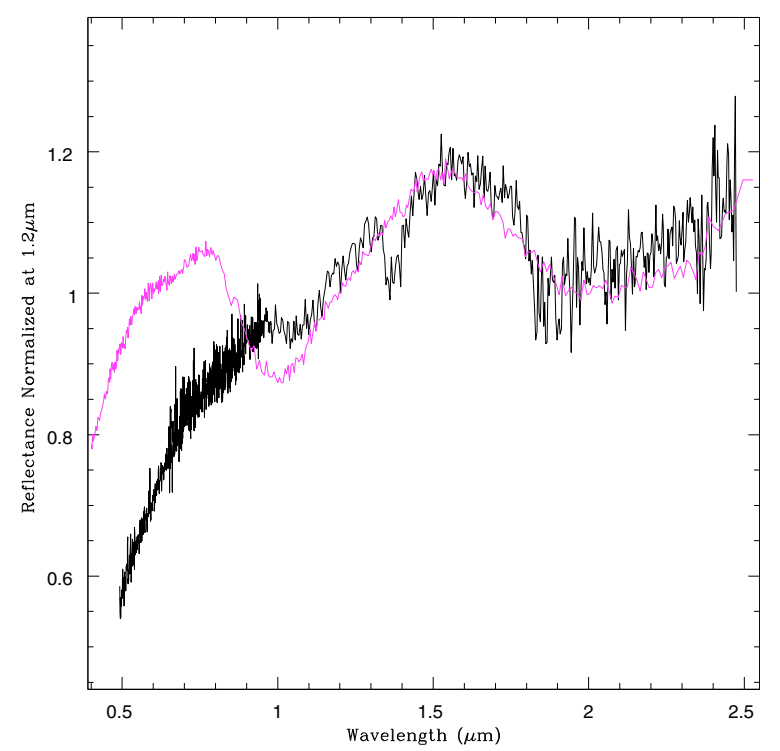

Fig. 4. Visible + NIR spectrum of (1400) Tirela compared to the spectrum of the mineral pigeonite (spectrum with the lower noise). The spectrum of the mineral has a stronger contrast in the visible with respect to the $1.0 \mu \mathrm{m}$ band, and does not fit the shape of the spectrum of Tirela.

know from studies on Earth, is unstable at low cooling rates, and is preserved in the original materials only if the cooling rates are high.

In a recent work, Sunshine et al. (2008) used the Hapke radiative transfer theory (Hapke 1993) to model the spectra of three L-type objects with an extremely large amount of CAI material $(30 \%)$ plus Mg-rich olivine. Other materials could also replace the CAI material in the nonlinear radiative model, such as chromium. Therefore the studies about the mineralogy of the $\mathrm{L} / \mathrm{Ld}$ classes are still inconclusive.

\section{Conclusions}

In this study we observed for the first time a significant amount of members of the Tirela family. The first classifications made for the family suggested that it could be the first D-type asteroid family in the main belt. Our analysis, however, indicates that the visible spectra of most of the family members belong to the taxonomic class Ld. One of these Ld-type objects, (1400) Tirela, was also observed in the near-infrared, and comparisons with minerals suggest similarities between the absorptions found in Tirela with those found in the mineral pigeonite. From the nature of an asteroid family and because all objects have the visible spectrum similar to the spectrum of Tirela or are even more suggestive for an $1.0 \mu \mathrm{m}$ band, we believe that they all (excluding the interloper B-type) share spectroscopic characteristics in the NIR. It is worth to emphasizing here the difficulties in observing those faint objects in the NIR, even though we have tried in our IRTF runs. Silicate bands are not present in D-type objects located out of the main belt (for Trojans, see Dotto 2006) or to cometary nuclei; therefore, we have no reason to believe that Tirela came from the outer regions of the SS. It probably was formed near its present location or even in the inner regions of the Solar System.

In the context of the Levison et al. (2008) work, the propensity of the D-type families predicted by these authors therefore contrasts with the present asteroid belt where no such families can be found. One solution of this problem is that the D-type 
families do not survive Gys because D-type objects are fragile and are eliminated by collisions with main-belt asteroids.

Finally, for most of the D-type objects in the asteroid belt, only the visible spectrum is available in the literature. We suggest that our understanding of the composition of some of these objects may be misleading, and these objects could instead represent mineralogies in the NIR that are completely different from our present understanding of surface composition of D-types, as we saw in the case of (1400) Tirela.

\section{Acknowledgements. Based on:}

- observations obtained at the Gemini Observatory, which is operated by the Association of Universities for Research in Astronomy, Inc., under a cooperative agreement with the NSF on behalf of the Gemini partnership: the National Science Foundation (United States), the Science and Technology Facilities Council (United Kingdom), the National Research Council (Canada), CONICYT (Chile), the Australian Research Council (Australia), CNPq (Brazil) and SECYT (Argentina)

- observations obtained with the Telescopio Nazionale Galileo (TNG), with a primary mirror of $3.58 \mathrm{~m}$, located at Roque de Los Muchachos Observatory (ORM).

- observations as Visiting Astronomer at the Infrared Telescope Facility, which is operated by the University of Hawaii under Cooperative Agreement no. NCC 5-538 with the National Aeronautics and Space Administration, Science Mission Directorate, Planetary Astronomy Program.

- plots in this paper were produced with SuperMongo plotting package http://www.astro.princeton.edu/ rhl/sm/. We thank Patricia Monger for her kind support for installing the software on new platforms.

\section{References}

Burbine, T. H., \& Binzel, R. P. 2002, Icarus, 159, 468

Bus, S. J., \& Binzel, R. P. 2002a, Icarus, 158, 146

Bus, S. J., \& Binzel, R. P. 2002b, Icarus, 158, 106

Carvano, J. M., Mothé-Diniz, T., \& Lazzaro, D. 2003, Icarus, 161, 356

Clark, R. N., Swayze, G. A., Livo, K. E., et al. 2003, J. Geophys. Res. (Planets), 108,5131

Doressoundiram, A., Barucci, M. A., Fulchignoni, M., \& Florczak, M. 1998, Icarus, 131, 15

Florczak, M., Barucci, M. A., Doressoundiram, A., et al. 1998, Icarus, 133, 233 Florczak, M., Lazzaro, D., Mothé-Diniz, T., Angeli, C. A., \& Betzler, A. S. 1999, A\&AS, 134, 463

Hapke, B. W. 1993, Theory of Reflectance and Emittance Spectroscopy (Cambridge University Press)

Hirayama, K. 1918, AJ, 31, 185

Hook, I. M., Jørgensen, I., Allington-Smith, J. R., et al. 2004, PASP, 116, 425

Lazzaro, D., Mothé-Diniz, T., Carvano, J. M., et al. 1999, Icarus, 142, 445

Lazzaro, D., Angeli, C. A., Carvano, J. M., et al. 2004, Icarus, 172, 179

Levison, H. F., Bottke, W. F., Gounelle, M., et al. 2008, Nature, submitted

Mothé-Diniz, T., \& Carvano, J. M. 2005, A\&A, 174, 54

Mothé-Diniz, T., Martino, M. D., Bendjoya, P., Doressoundiram, A., \& Migliorini, F. 2001, Icarus, 152, 117

Mothé-Diniz, T., Roig, F., \& Carvano, J. M. 2005, Icarus, 174, 54

Mothé-Diniz, T., Carvano, J. M., Bus, S., Duffardd, R., \& Burbine, T. 2008, Icarus, 195, 277

Nesvorný, D., Jedicke, R., Whiteley, R. J., \& Ivezić, Ž. 2005, Icarus, 173, 132

Pieters, C. M., \& Hiroi, T. 2004, in Lunar and Planetary Institute Conference Abstracts, 1720

Stoughton, C., Lupton, R. H., Bernardi, M., et al. 2002, AJ, 123, 485

Sunshine, J. M., Connolly, H. C., McCoy, T. J., Bus, S. J., \& La Croix, L. M. 2008, Science, 320, 514

Zappalà, V., Bendjoya, P., Cellino, A., Farinella, P., \& Froeschlé, C. 1995, Icarus, 116, 291 